\title{
EXISTENCE AND CONTINUOUS DEPENDENCE OF THE LOCAL SOLUTION OF NON-HOMOGENEOUS KdV-K-S EQUATION IN PERIODIC SOBOLEV SPACES
}

\section{YOLANDA SILVIA SANTIAGO AYALA and SANTIAGO CESAR ROJAS ROMERO}

Facultad de Ciencias Matemáticas

Universidad Nacional Mayor de San Marcos

Av. Venezuela Cdra. 34, Lima 01

Lima

Perú

e-mail: ysantiagoa@unmsm.edu.pe srojasr@unmsm.edu.pe (CC BY 3.0). 


\begin{abstract}
In this article, we prove that initial value problem associated to the nonhomogeneous KdV-Kuramoto-Sivashinsky (KdV-K-S) equation in periodic Sobolev spaces has a local solution in $[0, T]$ with $T>0$, and the solution has continuous dependence with respect to the initial data and the nonhomogeneous part of the problem. We do this in an intuitive way using Fourier theory and introducing a $C_{O}$-semigroup inspired by the work of Iorio [2] and Ayala and Romero [8].

Also, we prove the uniqueness solution of the homogeneous and nonhomogeneous KdV-K-S equation, using its dissipative property, inspired by the work of Iorio [2] and Ayala and Romero [9].
\end{abstract}

\title{
1. Introduction
}

First, we want to comment that from Theorem 3.1 in [7], we have that the KdV-K-S homogeneous problem is globally well posed and, in addition to the inequality (3.2) in [7], we have the continuous dependence of the solution of homogeneous problem. In this work, in Theorem 3.2, we will prove the existence and uniqueness of the local solution for the nonhomogeneous problem, and from inequality (3.8), we will get the continuous dependence of the solution with respect to the initial data and respect to the non-homogeneous part.

Thus, in both homogeneous and non-homogeneous cases, the estimatives are made from the explicit form of the solution, that is, by applying the Fourier transform to the respective equation.

Another result in this work is about the dissipative property of the homogeneous problem and some estimates of it, using differential calculus in $H_{\text {per }}^{s}$. This is included in Theorem 3.3 which we will develop in Subsection 3.2. So, using Theorem 3.3, we deduce the results of continuous dependence and uniqueness of solution for both homogeneous and non-homogeneous problems, respectively.

Finally, we obtain the continuous dependence and uniqueness of solution for the non-homogeneous case, applying the corresponding properties from [7]. 
We cite some works about KdV-K-S equation [1], [3] and for dissipative properties of systems [4].

Our article is organized as follows. In Section 2, we indicate the methodology used and cite the references used. In Section 3, we proved the main results.

Finally, in Section 4, we give the conclusions of our study.

\section{Methodology}

As a theoretical framework in this article we use the existence and regularity results of [7]. Also, we use the references [2], [6], [7], [8], [9], [10] and [5] for the Fourier theory in periodic Sobolev spaces, and differential and integral calculus in Banach spaces.

\section{Main Results}

First, using the Fourier transform, we will prove that the nonhomogeneous problem has a unique solution and it continuously depends respect to the initial data and the non-homogeneity.

Second, we will study the uniqueness of the solution for homogeneous case using another technique that involves the dissipative property of the problem.

Finally, we will remark two additional ways leading to the continuous dependence of the solution for the non-homogeneous problem.

\subsection{The non-homogeneous problem $\left(P_{c}^{F}\right)$ is locally well posed}

Theorem 3.1. Let $\phi \in H_{\text {per }}^{s}, s \in \mathbb{R}, \mu>0, F \in C\left([0, T], H_{\text {per }}^{s}\right)$, where $T>0,\{S(t)\}_{t \geq 0}$ the semigroup of class $C_{o}$ of homogeneous case $(F=0)$, introduced in the Theorem 3.2 from [3], and

$$
u_{p}(t):=\int_{0}^{t} S(t-\tau) F(\tau) d \tau
$$


then $u_{p} \in C\left([0, T], H_{\text {per }}^{s}\right) \cap C^{1}\left([0, T], H_{\text {per }}^{s-4}\right)$ and satisfies

$$
\mid \begin{aligned}
& \partial_{t} u_{p}+\partial_{x}^{3} u_{p}+\mu\left(\partial_{x}^{4} u_{p}+\partial_{x}^{2} u_{p}\right)=F(t) \in H_{\mathrm{per}}^{s-4} \\
& u_{p}(0)=0
\end{aligned}
$$

with the derivative given by

$$
\lim _{h \rightarrow 0}\left\|\frac{u_{p}(t+h)-u_{p}(t)}{h}+\partial_{x}^{3} u_{p}+\mu\left(\partial_{x}^{4} u_{p}+\partial_{x}^{2} u_{p}\right)-F(t)\right\|_{s-4}=0 .
$$

Proof. We remark that $S(t-\tau) F(\tau) \in H_{\text {per }}^{s}, \forall \tau \in(0, t)$ and $\tau \mapsto S(t-\tau) F(\tau)$ is continuous in $[0, t]$ then $\underbrace{\exists \int_{0}^{t} S(t-\tau) F(\tau) d \tau}_{u_{p}(t):=} \in H_{\mathrm{per}}^{s}$.

Now, we will prove $u_{p} \in C\left([0, T], H_{\text {per }}^{s}\right)$, that is, $\left\|u_{p}(t+h)-u_{p}(t)\right\|_{s} \rightarrow 0$ when $h \rightarrow 0$.

Let $h>0$

$$
\begin{aligned}
u_{p}(t+h)-u_{p}(t)= & \int_{0}^{t+h} S(t+h-\tau) F(\tau) d \tau-\int_{0}^{t} S(t-\tau) F(\tau) d \tau \\
= & \int_{0}^{t}\{S(t+h-\tau)-S(t-\tau)\} F(\tau) d \tau \\
& +\int_{t}^{t+h} S(t+h-\tau) F(\tau) d \tau
\end{aligned}
$$

taking the norm $\|\cdot\|_{s}$ we obtain

$$
\begin{aligned}
\left\|u_{p}(t+h)-u_{p}(t)\right\|_{s} \leq & \underbrace{\left\|\int_{0}^{t}\{S(t+h-\tau)-S(t-\tau)\} F(\tau) d \tau\right\|_{s}}_{I_{1}:=} \\
& +\underbrace{\left\|\int_{t}^{t+h} S(t+h-\tau) F(\tau) d \tau\right\|_{s}}_{I_{2}:=} .
\end{aligned}
$$


Using the $M$-Test of Weierstrass, we get

$$
\begin{aligned}
I_{1} & \leq \int_{0}^{t}\|S(t+h-\tau) F(\tau)-S(t-\tau) F(\tau)\|_{S} d \tau \\
& \leq \frac{\epsilon}{2 T} \int_{0}^{t} d \tau=\frac{\epsilon t}{2 T} \leq \frac{\epsilon T}{2 T}=\frac{\epsilon}{2}
\end{aligned}
$$

since $\exists \delta>0$ such that:

$$
\text { if }|h|<\delta \text {, then }\|S(t+h-\tau) F(\tau)-S(t-\tau) F(\tau)\|_{s}<\frac{\epsilon}{2 T}, \forall \tau \in(0, t) .
$$

Using the mean value Theorem in $H_{\text {per }}^{s}$, we obtain

$$
\frac{1}{h} \int_{t}^{t+h} S(t+h-\tau) F(\tau) d \tau \rightarrow S(0) F(t)=F(t)
$$

when $h \rightarrow 0$. Then

$$
\int_{t}^{t+h} S(t+h-\tau) F(\tau) d \tau=\underbrace{h}_{\rightarrow 0} \cdot \underbrace{\frac{1}{h} \int_{t}^{t+h} S(t+h-\tau) F(\tau) d \tau}_{\rightarrow F(t)} \rightarrow 0,
$$

when $h \rightarrow 0$. So, we have

$$
\left\|\int_{t}^{t+h} S(t+h-\tau) F(\tau) d \tau\right\|_{s} \rightarrow 0
$$

when $h \rightarrow 0$. That is, $I_{2}<\frac{\epsilon}{2}$ whenever $|h|<\delta^{*}$.

Therefore, $\left\|u_{p}(t+h)-u_{p}(t)\right\|_{s} \leq I_{1}+I_{2}<\epsilon$ whenever $|h|<\min \left\{\delta, \delta^{*}\right\}$.

From definition of $u_{p}(t)$ we have $u_{p}(0)=0$. 
Now, we will prove that $\exists \partial_{t} u_{p}(t)$ in $H_{\text {per }}^{s}$. In effect,

$$
\begin{aligned}
& \frac{u_{p}(t+h)-u_{p}(t)}{h} \\
& =\frac{1}{h}\left\{\int_{0}^{t+h} S(t+h-\tau) F(\tau) d \tau-\int_{0}^{t} S(t-\tau) F(\tau) d \tau\right\} \\
& =\frac{1}{h}\left\{\int_{0}^{t}\{S(t+h-\tau) F(\tau)-S(t-\tau) F(\tau)\} d \tau+\int_{t}^{t+h} S(t+h-\tau) F(\tau) d \tau\right\} \\
& =\frac{1}{h} \int_{0}^{t}\{S(t+h-\tau) F(\tau)-S(t-\tau) F(\tau)\} d \tau+\frac{1}{h} \int_{t}^{t+h} S(t+h-\tau) F(\tau) d \tau
\end{aligned}
$$

Using the mean value Theorem in $H_{\text {per }}^{s-4}$, we obtain

$$
\frac{1}{h} \int_{t}^{t+h} S(t+h-\tau) F(\tau) d \tau \rightarrow S(0) F(t)=F(t)
$$

when $h \rightarrow 0$.

Since

$$
\frac{S(t+h-\tau) F(\tau)-S(t-\tau) F(\tau)}{h}
$$

converges uniformly to $\partial_{t}\{S(t-\tau) F(\tau)\}$ in $H_{\text {per }}^{s-4}, \forall \tau \in[0, t]$, we obtain that

$$
\int_{0}^{t} \frac{S(t+h-\tau) F(\tau)-S(t-\tau) F(\tau)}{h} d \tau
$$

converges to $\int_{0}^{t} \partial_{t}\{S(t-\tau) F(\tau)\} d \tau$ when $h \rightarrow 0$. 
Now, we remark that $\partial_{t}\{S(t-\tau) F(\tau)\}=\left(-\partial_{x}^{3}-\mu\left(\partial_{x}^{4}+\partial_{x}^{2}\right)\right) S(t-\tau) F(\tau)$ in $H_{\text {per }}^{s-4}$. Since $\left(-\partial_{x}^{3}-\mu\left(\partial_{x}^{4}+\partial_{x}^{2}\right)\right)$ is a closed operator, then

$$
\int_{0}^{t}\left(-\partial_{x}^{3}-\mu\left(\partial_{x}^{4}+\partial_{x}^{2}\right)\right) S(t-\tau) F(\tau) d \tau=\left(-\partial_{x}^{3}-\mu\left(\partial_{x}^{4}+\partial_{x}^{2}\right)\right) \int_{0}^{t} S(t-\tau) F(\tau) d \tau
$$

Therefore,

$$
\int_{0}^{t} \partial_{t}\{S(t-\tau) F(\tau)\} d \tau=\left(-\partial_{x}^{3}-\mu\left(\partial_{x}^{4}+\partial_{x}^{2}\right)\right) \underbrace{\int_{0}^{t} S(t-\tau) F(\tau) d \tau}_{u_{p}(t):=} .
$$

That is,

$$
\int_{0}^{t} \frac{S(t+h-\tau) F(\tau)-S(t-\tau) F(\tau)}{h} d \tau \rightarrow\left(-\partial_{x}^{3}-\mu\left(\partial_{x}^{4}+\partial_{x}^{2}\right)\right) u_{p}(t),
$$

when $h \rightarrow 0$.

Finally, in $H_{\text {per }}^{s-4}$, using (3.3) and (3.4), we get

$$
\underbrace{\exists \lim _{h \rightarrow 0} \frac{u_{p}(t+h)-u_{p}(t)}{h}}_{\partial_{t} u_{p}(t)=}=\left(-\partial_{x}^{3}-\mu\left(\partial_{x}^{4}+\partial_{x}^{2}\right)\right) u_{p}(t)+F(t) .
$$

Using that $\left\|\partial_{x}^{m} f\right\|_{s-m} \leq\|f\|_{s}, \forall m \in Z^{+}, \forall f \in H_{\text {per }}^{s}$, we obtain

$$
\begin{aligned}
\left\|\partial_{t} u_{p}(t+h)-\partial_{t} u_{p}(t)\right\|_{s-4} \\
=\|\left(-\partial_{x}^{3}-\mu\left(\partial_{x}^{4}+\partial_{x}^{2}\right)\right) u_{p}(t+h)+F(t+h) \\
\quad-\left\{\left(-\partial_{x}^{3}-\mu\left(\partial_{x}^{4}+\partial_{x}^{2}\right)\right) u_{p}(t)+F(t) \|_{s-4}\right. \\
\leq\|F(t+h)-F(t)\|_{s-4}+\left\|\left(-\partial_{x}^{3}-\mu\left(\partial_{x}^{4}+\partial_{x}^{2}\right)\right)\left\{u_{p}(t+h)-u_{p}(t)\right\}\right\|_{s-4} \\
\leq\|F(t+h)-F(t)\|_{s-4}+\left\|-\partial_{x}^{3}\left\{u_{p}(t+h)-u_{p}(t)\right\}\right\|_{s-4} \\
\quad+\left\|-\mu \partial_{x}^{4}\left\{u_{p}(t+h)-u_{p}(t)\right\}\right\|_{s-4}+\left\|-\mu \partial_{x}^{2}\left\{u_{p}(t+h)-u_{p}(t)\right\}\right\|_{s-4}
\end{aligned}
$$




$$
\begin{aligned}
\leq & \|F(t+h)-F(t)\|_{s-4}+\left\|u_{p}(t+h)-u_{p}(t)\right\|_{s-1} \\
& +\mu\left\|u_{p}(t+h)-u_{p}(t)\right\|_{s}+\mu\left\|u_{p}(t+h)-u_{p}(t)\right\|_{s-2} .
\end{aligned}
$$

Since $u_{p}(t) \in H_{\text {per }}^{s} \subset H_{\text {per }}^{s-1} \subset H_{\text {per }}^{s-2}$, then

$$
\begin{aligned}
\| \partial_{t} u_{p}(t+ & h)-\partial_{t} u_{p}(t) \|_{s-4} \\
& \leq\|F(t+h)-F(t)\|_{s-4}+(1+2 \mu)\left\|u_{p}(t+h)-u_{p}(t)\right\|_{s} \rightarrow 0,
\end{aligned}
$$

when $h \rightarrow 0$.

Now, since $F$ is continuous in $H_{\text {per }}^{s-4}$ and $u_{p}$ is continuous in $H_{\text {per }}^{s}$, then $\partial_{t} u_{p}$ is continuous in $H_{\text {per }}^{s-4}$ for $t \in[0, T]$, that is, $\partial_{t} u_{p} \in C([0, T]$, $\left.H_{\mathrm{per}}^{s-4}\right)$.

Therefore, $u_{p} \in C\left([0, T], H_{\text {per }}^{s}\right) \cap C^{1}\left([0, T], H_{\text {per }}^{s-4}\right)$.

Theorem 3.2. Let $\phi \in H_{\text {per }}^{s}, s \in \mathbb{R}, \mu>0, F \in C\left([0, T], H_{\text {per }}^{s}\right)$, where $T>0$, and $\{S(t)\}_{t \geq 0}$ the semigroup of class $C_{o}$ of contraction in $H_{\mathrm{per}}^{s}$ as in Theorem 3.1, then

(1) The function

$$
u^{F}(t):=S(t) \phi+\underbrace{\int_{0}^{t} S(t-\tau) F(\tau) d \tau}_{u_{p}(t)=}, t \in[0, T]
$$

belongs to $C\left([0, T], H_{\mathrm{per}}^{s}\right) \cap C^{1}\left([0, T], H_{\mathrm{per}}^{s-4}\right)$ and

(2) $u^{F}(t)$ is the unique solution of

$$
\left(P_{c}^{F}\right) \mid \begin{aligned}
& u_{t}+u_{x x x}+\mu\left(u_{x x x x}+u_{x x}\right)=F(t) \in H_{\mathrm{per}}^{s-4}, \\
& u(0)=\phi
\end{aligned}
$$


with the derivative given by

$$
\lim _{h \rightarrow 0}\left\|\frac{u(t+h)-u(t)}{h}+u_{x x x}+\mu\left(u_{x x x x}+u_{x x}\right)-F(t)\right\|_{s-4}=0 .
$$

(3) Let $\psi_{j} \in H_{\mathrm{per}}^{s}, F_{j} \in C\left([0, T], H_{\mathrm{per}}^{s}\right), j=1,2$. The map $\psi \rightarrow u$ is continuous in the following sense. Let $u_{1}$ and $u_{2}$ the corresponding solutions to initial data $\psi_{1}$ and $\psi_{2}$, and with non-homogeneity $F_{1}$ and $F_{2}$, respectively. Then

$$
\begin{aligned}
&\left\|u_{1}(t)-u_{2}(t)\right\|_{s} \leq\left\|\psi_{1}-\psi_{2}\right\|_{s}+T\left\|F_{1}-F_{2}\right\|_{\infty, s-4}, t \in[0, T] \\
& \underbrace{\sup _{t \in[0, T]}\left\|u_{1}(t)-u_{2}(t)\right\|_{s}}_{\left\|u_{1}-u_{2}\right\|_{\infty, s}} \leq\left\|\psi_{1}-\psi_{2}\right\|_{s}+T\left\|F_{1}-F_{2}\right\|_{\infty, s-4}, \\
&\left\|\partial_{t} u_{1}(t)-\partial_{t} u_{2}(t)\right\|_{s-4} \leq(1+2 \mu)\left\|u_{1}(t)-u_{2}(t)\right\|_{s}+\left\|F_{1}-F_{2}\right\|_{\infty, s-4}, t \in[0, T], \\
& \leq(1+2 \mu)\left\|u_{1}-u_{2}\right\|_{\infty, s}+\left\|F_{1}-F_{2}\right\|_{\infty, s-4} \\
& \leq(1+2 \mu)\left\|\psi_{1}-\psi_{2}\right\|_{s}+[(1+2 \mu) T+1]\left\|F_{1}-F_{2}\right\|_{\infty, s-4},
\end{aligned}
$$

where we have used the notation

$$
\|h\|_{\infty, r}=\sup _{t \in[0, T]}\|h(t)\|_{r}, h \in C\left([0, T], H_{\mathrm{per}}^{r}\right) .
$$

Proof. We work the following steps:

(1) Let $u(t):=u^{F}(t)=S(t) \phi+u_{p}(t)$, we will prove that $u \in C([0, T]$, $\left.H_{\mathrm{per}}^{s}\right) \cap C^{1}\left([0, T], H_{\mathrm{per}}^{s-4}\right)$. In effect, as $S(\cdot) \phi \in C\left([0, T], H_{\mathrm{per}}^{s}\right)$ and $u_{p}(\cdot) \phi \in C\left([0, T], H_{\mathrm{per}}^{s}\right)$, then $u(\cdot)=S(\cdot) \phi+u_{p}(\cdot) \in C\left([0, T], H_{\mathrm{per}}^{s}\right)$. 
Moreover, as $S(\cdot) \phi \in C^{1}\left([0,+\infty), H_{\mathrm{per}}^{s-4}\right)$ and $u_{p}(\cdot) \in C^{1}\left([0, T], H_{\mathrm{per}}^{s-4}\right)$, then $u(\cdot)=S(\cdot) \phi+u_{p}(\cdot) \in C^{1}\left([0, T], H_{\text {per }}^{s-4}\right)$.

(2) We will prove that $u$ is the solution of $\left(P_{c}^{F}\right)$. In effect, we know that $\exists \partial_{t} S(t) \phi$ and $\exists \partial_{t} u_{p}(t)$ in $H_{\text {per }}^{s-4}$, then

$$
\begin{aligned}
\partial_{t} u(t)= & \partial_{t} \underbrace{S(t) \phi}_{u_{h}(t)=}+\partial_{t} u_{p}(t) \\
= & -\partial_{x}^{3} u_{h}(t)-\mu\left(\partial_{x}^{4} u_{h}(t)+\partial_{x}^{2} u_{h}(t)\right) \\
& -\partial_{x}^{3} u_{p}(t)-\mu\left(\partial_{x}^{4} u_{p}(t)+\partial_{x}^{2} u_{p}(t)\right)+F(t) \\
= & -\partial_{x}^{3}\left\{u_{h}(t)+u_{p}(t)\right\}-\mu\left(\partial_{x}^{4}\left\{u_{h}(t)+u_{p}(t)\right\}\right. \\
& \left.+\partial_{x}^{2}\left\{u_{h}(t)+u_{p}(t)\right\}\right)+F(t) \\
= & -\partial_{x}^{3} u(t)-\mu\left(\partial_{x}^{4} u(t)+\partial_{x}^{2} u(t)\right)+F(t)
\end{aligned}
$$

in $H_{\text {per }}^{s-4}$, where $u_{h}(\cdot)$ is solution of the homogeneous equation $\left(P_{c}\right)$.

(3) Also, $u(0)=u_{h}(0)+u_{p}(0)=\phi+0=\phi$.

(4) Let $\psi_{j} \in H_{\text {per }}^{s}$ and $F_{j} \in C\left([0, T], H_{\text {per }}^{s}\right)$ for $j=1,2$, then

$$
u_{j}(t)=S(t) \psi_{j}+\int_{0}^{t} S(t-\tau) F_{j}(\tau) d \tau
$$

is solution of $\left(P_{c}^{F_{j}}\right)$ with initial data $u_{j}(0)=S(0) \psi_{j}=\psi_{j}$, for $j=1,2$.

Then

$$
u_{1}(t)-u_{2}(t)=S(t)\left\{\psi_{1}-\psi_{2}\right\}+\int_{0}^{t} S(t-\tau)\left\{F_{1}(\tau)-F_{2}(\tau)\right\} d \tau
$$


From where we obtain, for $t<T$ :

$$
\begin{aligned}
\left\|u_{1}(t)-u_{2}(t)\right\|_{s} & \leq\left\|S(t)\left\{\psi_{1}-\psi_{2}\right\}\right\|_{2}+\int_{0}^{t}\left\|S(t-\tau)\left\{F_{1}(\tau)-F_{2}(\tau)\right\}\right\|_{s} d \tau \\
& \leq\left\|\psi_{1}-\psi_{2}\right\|_{s}+\int_{0}^{t}\left\|F_{1}(\tau)-F_{2}(\tau)\right\|_{s} d \tau \\
& \leq\left\|\psi_{1}-\psi_{2}\right\|_{s}+\sup _{\tau \in[0, T]}\left\|F_{1}(\tau)-F_{2}(\tau)\right\|_{s} \int_{0}^{t} d \tau \\
& \leq\left\|\psi_{1}-\psi_{2}\right\|_{s}+T \cdot \sup _{\tau \in[0, T]}\left\|F_{1}(\tau)-F_{2}(\tau)\right\|_{s} .
\end{aligned}
$$

Therefore,

$$
\sup _{t \in[0, T]}\left\|u_{1}(t)-u_{2}(t)\right\|_{s} \leq\left\|\psi_{1}-\psi_{2}\right\|_{s}+T \cdot \sup _{\tau \in[0, T]}\left\|F_{1}(\tau)-F_{2}(\tau)\right\|_{s} .
$$

(5) On the other hand, in $H_{\text {per }}^{s-4}$, we have

$$
\begin{aligned}
\partial_{t} u_{j}(t) & =\partial_{t} u_{h, j}(t)+\partial_{t} u_{p, j} \\
& =\left[-\partial_{x}^{3}-\mu\left(\partial_{x}^{4}+\partial_{x}^{2}\right)\right] u_{h, j}+\left[-\partial_{x}^{3}-\mu\left(\partial_{x}^{4}+\partial_{x}^{2}\right)\right] u_{p, j}+F_{j}(t) \\
& =\left[-\partial_{x}^{3}-\mu\left(\partial_{x}^{4}+\partial_{x}^{2}\right)\right] u_{j}+F_{j}(t),
\end{aligned}
$$

for $j=1,2$. So,

$$
\partial_{t} u_{1}(t)-\partial_{t} u_{2}(t)=\left[-\partial_{x}^{3}-\mu\left(\partial_{x}^{4}+\partial_{x}^{2}\right)\right]\left\{u_{1}-u_{2}\right\}+\left\{F_{1}(t)-F_{2}(t)\right\} .
$$

Taking norm, we obtain

$$
\begin{aligned}
\left\|\partial_{t} u_{1}(t)-\partial_{t} u_{2}(t)\right\|_{s-4}= & \left\|\left[-\partial_{x}^{3}-\mu\left(\partial_{x}^{4}+\partial_{x}^{2}\right)\right]\left\{u_{1}-u_{2}\right\}+\left\{F_{1}(t)-F_{2}(t)\right\}\right\|_{s-4} \\
\leq & \left\|\partial_{x}^{3}\left\{u_{1}-u_{2}\right\}\right\|_{s-4}+\mu\left\|\partial_{x}^{4}\left\{u_{1}-u_{2}\right\}\right\|_{s-4} \\
& +\mu\left\|\partial_{x}^{2}\left\{u_{1}-u_{2}\right\}\right\|_{s-4}+\left\|F_{1}(t)-F_{2}(t)\right\|_{s-4} .
\end{aligned}
$$


Using $\left\|\partial_{x}^{m} \psi\right\|_{s-m} \leq\|\psi\|_{s}$, for $m=1,2,3$ and $H_{\text {per }}^{s} \subset H_{\text {per }}^{s-1} \subset H_{\text {per }}^{s-2}$, we get

$$
\begin{aligned}
\left\|\partial_{t} u_{1}(t)-\partial_{t} u_{2}(t)\right\|_{s-4} \leq & \left\|u_{1}-u_{2}\right\|_{s-1}+\mu\left\|u_{1}-u_{2}\right\|_{s} \\
& +\mu\left\|u_{1}-u_{2}\right\|_{s-2}+\left\|F_{1}(t)-F_{2}(t)\right\|_{s-4} \\
& \leq(1+2 \mu)\left\|u_{1}-u_{2}\right\|_{s}+\left\|F_{1}(t)-F_{2}(t)\right\|_{s-4} \\
& \leq(1+2 \mu)\left\|u_{1}(t)-u_{2}(t)\right\|_{s}+\sup _{t \in[0, T]}\left\|F_{1}(t)-F_{2}(t)\right\|_{s-4} \\
& \leq(1+2 \mu)\left\|u_{1}-u_{2}\right\|_{\infty, s}+\left\|F_{1}-F_{2}\right\|_{\infty, s-4} \\
& \leq(1+2 \mu)\left\|\psi_{1}-\psi_{2}\right\|_{s}+[(1+2 \mu) T+1]\left\|F_{1}-F_{2}\right\|_{\infty, s-4} .
\end{aligned}
$$

Remark 3.1. Inequality (3.8) says that the solution of the nonhomogeneous problem $\left(P_{c}^{F}\right)$ continuously depends on the initial data and the non-homogeneity $F$.

Corollary 3.1. $\left(P_{c}^{F}\right)$ has a unique solution.

Proof. This follows by applying inequality (3.8) with $\psi_{1}=\psi_{2}$ and $F_{1}=F_{2}$.

Corollary 3.2. The unique solution of $\left(P_{c}^{F}\right)$ is

$$
\begin{aligned}
u^{F}(t, x)= & \sum_{k=-\infty}^{+\infty} e^{-\left\{-i k^{3}+\mu\left(k^{2}-1\right) k^{2}\right\} t} \widehat{\phi}(k) e^{i k x} \\
& +\int_{0}^{t} \sum_{k=-\infty}^{+\infty} e^{-\left\{-i k^{3}+\mu\left(k^{2}-1\right) k^{2}\right\}(t-\tau)} \widehat{F}(k, \tau) e^{i k x} d \tau .
\end{aligned}
$$




\subsection{Dissipative property of the homogeneous problem}

Let $\mu>0, s \in \mathbb{R}$ and the homogeneous problem

$$
\left(P_{c}\right) \mid \begin{aligned}
& w \in C\left([0, \infty), H_{\mathrm{per}}^{s}\right) \cap C^{1}\left([0, \infty), H_{\mathrm{per}}^{s-4}\right) \\
& \partial_{t} w+\partial_{x}^{3} w+\mu\left(\partial_{x}^{4} w+\partial_{x}^{2} w\right)=0 \in H_{\mathrm{per}}^{s-4} \\
& w(0)=\phi \in H_{\mathrm{per}}^{s} .
\end{aligned}
$$

Theorem 3.3. Let $w$ the solution of $\left(P_{c}\right)$ with initial data $\phi \in H_{\mathrm{per}}^{s}$, then we obtain the following results:

(1) $\partial_{t}\|w(t)\|_{s-4}^{2}=-2 \mu<\partial_{x}^{2} w(t)+\partial_{x}^{4} w(t), w(t)>_{s-4} \leq 0$.

(2) $\|w(t)\|_{s-4} \leq\|\phi\|_{s-4} \leq\|\phi\|_{s}, t \geq 0$.

Proof. As $H_{\text {per }}^{s} \subset H_{\text {per }}^{s-4}$ then the following expressions are well defined: $\left\langle\partial_{t} w, w>_{s-4}\right.$ and $<w, \partial_{t} w>_{s-4}$.

So, we have

$$
\begin{aligned}
\partial_{t}\|w(t)\|_{s-4}^{2} & =\partial_{t}<w(t), w(t)>_{s-4} \\
& =<\partial_{t} w(t), w(t)>_{s-4}+<w(t), \partial_{t} w(t)>_{s-4} \\
& =2 \operatorname{Re}<\partial_{t} w(t), w(t)>_{s-4} .
\end{aligned}
$$

For the other hand,

$$
\begin{aligned}
<\partial_{x}^{2} w+\partial_{x}^{4} w, w>_{s-4} & =2 \pi \sum_{k=-\infty}^{+\infty}\left(1+k^{2}\right)^{s-4}\left\{\partial_{x}^{2} \widehat{w+\partial_{x}^{4} w}(k)\right\} \overline{\widehat{w}(k)} \\
& =2 \pi \sum_{k=-\infty}^{+\infty}\left(1+k^{2}\right)^{s-4} \underbrace{k^{2}\left(k^{2}-1\right)}_{M(k):=} \widehat{w}(k) \overline{\widehat{w}(k)} \\
& =2 \pi \sum_{k=-\infty}^{+\infty}\left(1+k^{2}\right)^{s-4} M(k)|\widehat{w}(k)|^{2} .
\end{aligned}
$$


We remark that the series in (3.13) is convergent since $M(k) \leq k^{4} \leq$ $\left(k^{2}\right)^{4} \leq\left(1+k^{2}\right)^{4}, \forall k \in Z$ and $w(t) \in H_{\mathrm{per}}^{s}$.

Moreover, as

$$
M(k)=\left\{\begin{array}{l}
0, \text { if } k \in\{-1,0,1\}, \\
k^{2}\left(k^{2}-1\right)>0, \text { if } k \in Z-\{-1,0,1\},
\end{array}\right.
$$

and $M(k) \geq 12, \forall k \in Z-\{-1,0,1\}$, then the convergent series (3.13) is positive.

That is,

$$
<\partial_{x}^{2} w+\partial_{x}^{4} w, w>_{s-4} \geq 0
$$

So, we have

$$
-<\partial_{x}^{2} w+\partial_{x}^{4} w, w>_{s-4} \leq 0
$$

Also, we obtain

$$
\begin{aligned}
\left.<\partial_{x}^{3} w, w\right\rangle_{s-4} & =2 \pi \sum_{k=\infty}^{+\infty}\left(1+k^{2}\right)^{s-4} \widehat{\partial_{x}^{3} w}(k) \cdot \overline{\widehat{w}(k)} \\
& =2 \pi \sum_{k=\infty}^{+\infty}\left(1+k^{2}\right)^{s-4}(i k)^{3} \widehat{w}(k) \cdot \overline{\hat{w}(k)} \\
& =-\underbrace{i \underbrace{2 \pi \sum_{k}^{+\infty}\left(1+k^{2}\right)^{s-4} k^{3}|\widehat{w}(k)|^{2}}_{k=-\infty}}_{b:=} .
\end{aligned}
$$

Now, we will prove that the series of the equality (3.15) is convergent. In effect, using the inequality: $|k|^{3} \leq|k|^{8}=\left(|k|^{2}\right)^{4} \leq\left(1+k^{2}\right)^{4}$ and $w(t) \in H_{\text {per }}^{s}$, we have 


$$
\begin{aligned}
\left.\left|\sum_{k=-\infty}^{+\infty}\left(1+k^{2}\right)^{s-4} k^{3}\right| \hat{w}(k)\right|^{2} \mid & \leq \sum_{k=-\infty}^{+\infty}\left(1+k^{2}\right)^{s-4}|k|^{3}|\widehat{w}(k)|^{2} \\
& \leq \sum_{k=-\infty}^{+\infty}\left(1+k^{2}\right)^{s-4}\left(1+k^{2}\right)^{4}|\widehat{w}(k)|^{2} \\
& =\sum_{k=-\infty}^{+\infty}\left(1+k^{2}\right)^{s}|\widehat{w}(k)|^{2}=\frac{1}{2 \pi}\|w(t)\|_{s}^{2}<\infty .
\end{aligned}
$$

Then the series is convergent, that is,

$$
<\partial_{x}^{3} w, w>_{s-4}=-i b \text {, with } b \in \mathbb{R} .
$$

From (3.12), using $\partial_{t} w=-\mu\left(\partial_{x}^{2} w+\partial_{x}^{4} w\right)-\partial_{x}^{3} w, \mu>0$, the inequality (3.14) and the equality (3.16), we get

$$
\begin{aligned}
\partial_{t}\|w(t)\|_{s-4}^{2}= & 2 \operatorname{Re}<\partial_{t} w(t), w(t)>_{s-4} \\
= & 2 \operatorname{Re}<-\mu\left(\partial_{x}^{2} w(t)+\partial_{x}^{4} w(t)\right)-\partial_{x}^{3} w(t), w(t)>_{s-4} \\
= & 2 \mu \operatorname{Re}\left\{-<\partial_{x}^{2} w(t)+\partial_{x}^{4} w(t), w(t)>_{s-4}\right\} \\
& -2 \operatorname{Re}<\partial_{x}^{3} w(t), w(t)>_{s-4} \\
= & -2 \mu \operatorname{Re}<\partial_{x}^{2} w(t)+\partial_{x}^{4} w(t), w(t)>_{s-4}-0 \\
= & -2 \mu \operatorname{Re}<\partial_{x}^{2} w(t)+\partial_{x}^{4} w(t), w(t)>_{s-4} \leq 0 .
\end{aligned}
$$

Therefore, $\|w(t)\|_{s-4}^{2}$ is not increasing. Then, $\|w(t)\|_{s-4}^{2} \leq\|w(0)\|_{s-4}^{2}, \forall t \geq 0$.

As $\left(\|w(t)\|_{s-4}-\|w(0)\|_{s-4}\right)\left(\|w(t)\|_{s-4}+\|w(0)\|_{s-4}\right) \leq 0$, we have

$$
\|w(t)\|_{s-4} \leq\|w(0)\|_{s-4} \leq\|w(0)\|_{s}, \forall t \geq 0 .
$$

That is, $\|w(t)\|_{s-4} \leq\|\phi\|_{s-4} \leq\|\phi\|_{s}, \forall t \geq 0$. 
Corollary 3.3 (Continuous dependence of the solution of $\left(P_{c}\right)$ ). Let $u$ and $v$ solutions of $\left(P_{c}\right)$ with initial data $\phi_{1}$ and $\phi_{2}$ in $H_{\mathrm{per}}^{s}$, respectively. Then the following assertions hold:

$\partial_{t}\|u(t)-v(t)\|_{s-4}^{2}=-2 \mu<\partial_{x}^{2}\{u(t)-v(t)\}+\partial_{x}^{4}\{u(t)-v(t)\}, u(t)-v(t)>_{s-4} \leq 0$,

and

$$
\|u(t)-v(t)\|_{s-4} \leq\left\|\phi_{1}-\phi_{2}\right\|_{s-4} \leq\left\|\phi_{1}-\phi_{2}\right\|_{s}, \quad t \geq 0 .
$$

Proof. Define $w:=u-v$ then $w$ satisfies:

$$
\mid \begin{aligned}
& \partial_{t} w+\partial_{x}^{3} w+\mu\left\{\partial_{x}^{2} w+\partial_{x}^{4} w\right\}=0 \\
& w(0)=\phi_{1}-\phi_{2}
\end{aligned}
$$

We conclude using the Theorem 3.3.

Corollary 3.4 (Uniqueness of solution of $\left(P_{c}\right)$ ). $\left(P_{c}\right)$ has a unique solution.

Proof. In effect, let $u$ and $v$ solutions of $\left(P_{c}\right)$ with the same initial data, that is, $\phi_{1}=\phi_{2}=\phi$.

From (3.17), we obtain $\|u(t)-v(t)\|_{s-4} \leq\|0\|_{s}=0$. Then, $\|u(t)-v(t)\|_{s-4}=0$. So, $u(t)=v(t), \forall t \geq 0$, that is, $u=v$.

\subsection{Continuous dependence and uniqueness solution of $\left(P_{c}^{F}\right)$ by} Theorem 3.3

The Theorem 3.3 will allow us to prove the following results.

Corollary 3.5 (Continuous dependence of the solution of $\left(P_{c}^{F}\right)$ ). Let $u$ and $v$ solutions of $\left(P_{c}^{F}\right)$ with initial data $\phi_{1}$ and $\phi_{2}$ in $H_{\mathrm{per}}^{s}$, respectively. Then $u-v$ is solution of $\left(P_{c}\right)$ with initial data $\phi_{1}-\phi_{2}$ and satisfies: 
$\partial_{t}\|u(t)-v(t)\|_{s-4}^{2}=-2 \mu<\partial_{x}^{2}\{u(t)-v(t)\}+\partial_{x}^{4}\{u(t)-v(t)\}, u(t)-v(t)>_{s-4} \leq 0$,

and

$$
\|u(t)-v(t)\|_{s-4} \leq\left\|\phi_{1}-\phi_{2}\right\|_{s-4} \leq\left\|\phi_{1}-\phi_{2}\right\|_{s} .
$$

Proof. It is an immediate consequence from Theorem 3.3.

Corollary 3.6 (Uniqueness solution of $\left.\left(P_{c}^{F}\right)\right) .\left(P_{c}^{F}\right)$ has a unique solution.

Proof. In effect, let $u$ and $v$ solutions of $\left(P_{c}^{F}\right)$ with the same initial data, that is $\phi_{1}=\phi_{2}=\phi \in H_{\text {per }}^{s}$, then using the Corollary 3.5, we have $u-v$ is solution of $\left(P_{c}\right)$ with initial data 0 and $\|u(t)-v(t)\|_{s-4} \leq\|0\|_{s}=0$.

Therefore, $\|u(t)-v(t)\|_{s-4}=0$. Thus, $u(t)=v(t), \forall t \geq 0$, that is, $u=v$.

\subsection{Continuous dependency and uniqueness solution of $\left(P_{c}^{F}\right)$ through results from [7]}

In this subsection, we will use results from [7] to prove the following corollary:

Corollary 3.7 (Continuous dependency of the solution of $\left(P_{c}^{F}\right)$ ). Let $u$ and $v$ solutions of $\left(P_{c}^{F}\right)$ with initial data $\phi_{1}$ and $\phi_{2}$ in $H_{\mathrm{per}}^{s}$, respectively. Then $u-v$ is solution of $\left(P_{c}\right)$ with initial data $\phi_{1}-\phi_{2}$ and satisfies:

$$
\begin{gathered}
\|u(t)-v(t)\|_{s} \leq\left\|\phi_{1}-\phi_{2}\right\|_{s}, t \in[0, T], \\
\|u(t)-v(t)\|_{r} \leq C\left\|\phi_{1}-\phi_{2}\right\|_{s}, \forall r \in \mathbb{R}, t \in(0, T] .
\end{gathered}
$$


Proof. From [7]: inequality (3.2) and inequality of Theorem 3.1, we conclude.

Remark 3.2. As a consequence of the Corollary 3.7, taking $\phi_{1}=\phi_{2}$, we obtain the uniqueness of solution of the problem $\left(P_{c}^{F}\right)$.

\section{Conclusion}

From our study of the KdV-K-S equation in periodic Sobolev spaces, for the homogeneous $\left(P_{c}\right)$ and non-homogeneous $\left(P_{c}^{F}\right)$ case, we have obtained the following results:

(1) In Theorem 3.2, using the Fourier transform, we proved that the non-homogeneous problem $\left(P_{c}^{F}\right)$ is locally well posed in compacts, obtaining continuous dependence with respect to the initial data and the non-homogeneity.

(2) We showed the dissipative property of the homogeneous problem, where an estimate for the norm of global solution was obtained, which allowed us to deduce the continuous dependence (with respect to the initial data) and uniqueness solution of $\left(P_{c}\right)$.

(3) We prove the continuous dependence respect to the initial data, and uniqueness solution of $\left(P_{c}^{F}\right)$ by using Theorem 3.3.

(4) We prove the continuous dependence respect to the initial data, and uniqueness solution of $\left(P_{c}^{F}\right)$ by using results from [7].

(5) In this paper, we have obtained results that can be applied to the KdV-K-S models with two parameters, and these promote the analysis of the corresponding convergence. 


\section{References}

[1] H. A. Biagioni, J. L. Bona, R. Iorio and M. Scialom, On the Korteweg-de VriesKuramoto-Sivashinsky equation, Advances in Differential Equations 1(1) (1996), $1-20$.

[2] R. J. Iorio, Fourier Analysis and Partial Differential Equations, Cambridge University, 2002.

[3] P. Guzman, S. Marx and E. Cerpa, Stabilization of the linear Kuramoto-Sivashinsky equation with a delayed boundary control, IFAC Papers Online 52(02) (2019), 70-75.

[4] Z. Liu and S. Zheng, Semigroups Associated with Dissipative System, Chapman Hall/CRC, 1999.

[5] H. K. Pathak, An Introduction to Nonlinear Analysis and Fixed Point Theory, Springer, Singapur, 2018.

[6] Yolanda Santiago Ayala, Santiago Rojas Romero and Teófanes Quispe Mendez, Espacios de sobolev periódico y unproblema de Cauchy asociado a un modelo de ondas en un fluido viscoso, Theorema, Segunda Epoca 3(04) (2016), 7-23.

[7] Yolanda Santiago Ayala and Santiago Rojas Romero, Regularity and well-posedness of a problem to one parameter and its behavior at the limit, Bulletin of the Allahabad Mathematical Society 32(02) (2017), 207-230.

[8] Yolanda Santiago Ayala and Santiago Rojas Romero, Existencia y regularidad de solución de la ecuación del calor en espacios de Sobolev periódico, Selecciones Matemáticas 06(1) (2019), 49-65.

DOI: http://dx.doi.org/10.17268/sel.mat.2019.01.08

[9] Yolanda Santiago Ayala and Santiago Rojas Romero, Unicidad de solución de la ecuación del calor en espacios de Sobolev periódico, Selecciones Matemáticas 07(1) (2020), 172-175.

DOI: http://dx.doi.org/10.17268/sel.mat.2020.01.16

[10] D. Stuart, Partial Differential Equations Example Sheet 2, 2014.

http://www.damtp.cam.ac.uk/user/examples/D15b.pdf 